PROCEEDINGS OF THE

AMERICAN MATHEMATICAL SOCIETY

Volume 134, Number 11, November 2006, Pages 3405-3415

S 0002-9939(06)08354-7

Article electronically published on May 8, 2006

\title{
RECONSTRUCTIONS OF DISTANCES BY ENERGY FORMS
}

\author{
SHIN-ICHI OHTA
}

(Communicated by Richard A. Wentworth)

\begin{abstract}
We prove that, if a metric measure space admits a stratification so that each stratum satisfies the strong doubling condition, then the intrinsic distance induced from the Cheeger-type energy form coincides with the original distance. In other words, we can reconstruct the distance function by the Cheeger-type energy form. We also observe that this reconstruction does not work for the Korevaar-Schoen-type energy form.
\end{abstract}

\section{INTRODUCTION}

The theory of Sobolev spaces for functions on an arbitrary metric measure space is making remarkable progress in recent years (see [C], [HK], [He, $\mathrm{KoSc}$, etc.). There the Sobolev space is defined as a space of functions with finite energies, and there are several definitions of energy forms on a metric measure space. Among them, in this article, we shall consider Cheeger's and Korevaar and Schoen's definitions $([\mathrm{C}],[\mathrm{KoSc})$, and intend to reveal the difference between them from the geometric point of view.

Our main theorem (Theorem 5.2) asserts that, if a metric measure space admits a stratification so that each stratum satisfies the strong doubling condition (in the sense of Ranjbar-Motlagh [R2]), then the intrinsic distance defined by using the Cheeger-type energy form coincides with the original distance. Here the strong doubling condition (Definition 5.1) can be regarded as a generalization of Measure Contraction Property in $[\mathrm{S}$ as well as the weak measure contraction property of Bishop-Gromov type in [KuSh, and the intrinsic distance (Definition 4.1) is defined as in $[\mathrm{BM}]$. The coincidence between the original distance and the intrinsic distance induced from the canonical Dirichlet form is known for Riemannian manifolds and, more generally, for Alexandrov spaces with lower curvature bounds ([KMS, Theorem 7.1]).

One aspect of the theorem is that we can reconstruct the distance function by using the energy form, and another aspect is that we can distinguish metric spaces by comparing the energy forms on them (Corollary 6.1). We will observe that the analogue is not true for the Korevaar-Schoen-type energy form in the case of

Received by the editors November 17, 2004 and, in revised form, May 25, 2005.

2000 Mathematics Subject Classification. Primary 58C05, 53C60.

Key words and phrases. Intrinsic distance, Cheeger-type energy form, Korevaar-Schoen-type energy form, strong doubling condition, geodesic bicombing.

This work was partially supported by the Grant-in-Aid for Scientific Research for Young Scientists (B) 16740034 from the Ministry of Education, Culture, Sports, Science and Technology, Japan.

(c)2006 American Mathematical Society Reverts to public domain 28 years from publication 
Banach spaces ( $\sqrt{6})$. Thus it seems that the Korevaar-Schoen-type energy form is suitable when we consider Riemannian spaces such as Alexandrov spaces, rather than Finsler spaces.

\section{Preliminaries for the Cheeger-type energy form}

This section is devoted to recalling the definition and some fundamental properties of the Cheeger-type energy form. See [C] for details. Throughout this article, let $\left(X, d_{X}\right)$ be a metric space and let $\mu$ be a Borel regular measure on $X$ such that $0<\mu(B(x, r))<\infty$ holds for all $x \in X$ and $r>0$. Here $B(x, r)$ denotes the open ball with center $x$ and radius $r$. For real numbers $a, b \in \mathbb{R}$, we set $a \wedge b:=\min \{a, b\}$ and $a \vee b:=\max \{a, b\}$. We will use some terminologies on Dirichlet forms (with quotation marks '...'); consult [FOT] for them.

Following the idea of Heinonen and Koskela [HeK], a Borel measurable function $g: X \longrightarrow[0, \infty]$ is called an upper gradient for a function $f: X \longrightarrow \mathbb{R}$ if, for any unit speed curve $\gamma:[0, l] \longrightarrow X$, we have

$$
|f(\gamma(0))-f(\gamma(l))| \leq \int_{0}^{l} g(\gamma(t)) d t
$$

Definition 2.1 (Cheeger-type energy form). For $p \in(1, \infty)$ and $f \in L^{p}(X)$, we define the Cheeger-type p-energy $E_{p}^{C}(f)$ of $f$ by

$$
E_{p}^{C}(f):=\inf _{\left\{\left(f_{i}, g_{i}\right)\right\}_{i=1}^{\infty}} \liminf _{i \rightarrow \infty}\left|g_{i}\right|_{L^{p}}^{p}
$$

where the infimum is taken over all sequences $\left\{\left(f_{i}, g_{i}\right)\right\}_{i=1}^{\infty}$ satisfying that $f_{i} \rightarrow f$ in $L^{p}(X)$ as $i \rightarrow \infty$ and that $g_{i}$ is an upper gradient for $f_{i}$ for each $i$. The Cheeger-type $(1, p)$-Sobolev space $H^{1, p}(X)$ is defined as a space

$$
H^{1, p}(X):=\left\{f \in L^{p}(X) \mid E_{p}^{C}(f)<\infty\right\}
$$

equipped with a norm $|f|_{H^{1, p}}:=|f|_{L^{p}}+E_{p}^{C}(f)^{1 / p}$.

We remark that the functions $f_{i}, g_{i} \in L^{p}(X)$ in the definition of $E_{p}^{C}$ is defined on the entire $X$, in other words, we actually consider some representatives of them. It is clear by definition that $E_{p}^{C}$ is 'Markovian'. Indeed, for any $f \in H^{1, p}(X)$, we have $E_{p}^{C}(0 \vee f \wedge 1) \leq E_{p}^{C}(f)$. It is also known that $E_{p}^{C}$ is 'closed' in the sense that $\left(H^{1, p}(X),|\cdot|_{H^{1, p}}\right)$ is complete ([C, Theorem 2.7]).

Remark 2.2. In general, the Cheeger-type 2-energy form $E_{2}^{C}$ is not necessarily bilinear in the sense that the symmetric form $\mathcal{E}: H^{1,2}(X) \times H^{1,2}(X) \longrightarrow \mathbb{R}$ defined by

$$
\mathcal{E}\left(f_{1}, f_{2}\right):=\frac{1}{4}\left\{E_{2}^{C}\left(f_{1}+f_{2}\right)-E_{2}^{C}\left(f_{1}-f_{2}\right)\right\}
$$

is not bilinear (see [0]). In particular, $\mathcal{E}$ is not actually a Dirichlet form. However, it is at the heart of our reconstruction. Compare this with the Korevaar-Schoen-type energy form which will be defined in $\$ 6$,

A function $g \in L^{p}(X)$ is called a generalized upper gradient for $f \in H^{1, p}(X)$ if there exist sequences $\left\{f_{i}\right\}_{i=1}^{\infty}$ and $\left\{g_{i}\right\}_{i=1}^{\infty}$ in $L^{p}(X)$ such that $f_{i} \rightarrow f$ and $g_{i} \rightarrow g$ in $L^{p}(X)$ as $i \rightarrow \infty$, respectively, and that $g_{i}$ is an upper gradient for $f_{i}$ for each $i$. By definition, it clearly holds that $E_{p}^{C}(f) \leq|g|_{L^{p}}^{p}$. A generalized upper gradient $g \in L^{p}(X)$ for $f \in H^{1, p}(X)$ is said to be minimal if it satisfies $|g|_{L^{p}}^{p}=E_{p}^{C}(f)$. 
Theorem 2.3 ([C] Theorem 2.10]). For any $f \in H^{1, p}(X)$, there exists a unique minimal generalized upper gradient $g \in L^{p}(X)$ for $f$.

We denote by $g_{f, p} \in L^{p}(X)$ the unique minimal generalized upper gradient for $f \in H^{1, p}(X)$.

Proposition 2.4 ('Strong locality', C, Corollary 2.25]). For functions $f_{1}, f_{2} \in$ $H^{1, p}(X)$ and a real number $a \in \mathbb{R}$, if $f_{1}=f_{2}+a$ holds a.e. on a measurable set $A \subset X$, then we have $g_{f_{1}, p}=g_{f_{2}, p}$ a.e. on $A$.

We also state two more known properties for later use.

Lemma 2.5 ([C, Lemma 1.7]). Let $g_{1}$ and $g_{2}$ be upper gradients for functions $f_{1}$ and $f_{2}$, respectively. Then, for any $\varepsilon>0$, the function $g_{1}\left(\left|f_{2}\right|+\varepsilon\right)+\left(\left|f_{1}\right|+\varepsilon\right) g_{2}$ is an upper gradient for $f_{1} f_{2}$.

Theorem 2.6 ([C], Theorem 2.5]). Let $f \in H^{1, p}(X)$ and $\left\{f_{i}\right\}_{i=1}^{\infty} \subset H^{1, p}(X)$ be a sequence such that $f_{i} \rightarrow f$ in $L^{p}(X)$ as $i$ tends to $\infty$. Then we have $E_{p}^{C}(f) \leq$ $\liminf _{i \rightarrow \infty} E_{p}^{C}\left(f_{i}\right)$.

\section{Regularity}

In this section, we show the 'regularity' of $E_{p}^{C}$ under some appropriate assumptions on $X$. Recall that we always assume that $1<p<\infty$.

Definition 3.1 (Doubling condition). A metric measure space $\left(X, d_{X}, \mu\right)$ is said to satisfy the (local) doubling condition if there exist constants $C_{D}=C_{D}(X) \geq 1$ and $R_{D}=R_{D}(X)>0$ such that

$$
\mu(B(x, 2 r)) \leq C_{D} \mu(B(x, r))
$$

holds for every $x \in X$ and $r \in\left(0, R_{D}\right]$.

Definition 3.2 (Poincaré inequality). A metric measure space $\left(X, d_{X}, \mu\right)$ is said to satisfy the (local) weak Poincaré inequality of type $(1, p)$ if there exist constants $C_{P}=C_{P}(X) \geq 1, R_{P}=R_{P}(X)>0$, and $\Lambda=\Lambda(X) \geq 1$ such that we have

$$
f_{B(x, r)}\left|f-f_{B(x, r)} f d \mu\right| d \mu \leq C_{P} r\left(f_{B(x, \Lambda r)}\left(g_{f, p}\right)^{p} d \mu\right)^{1 / p}
$$

for all $x \in X, r \in\left(0, R_{P}\right]$, and for all $f \in H^{1, p}(B(x, \Lambda r))$.

As usual, for a measurable set $A \subset X$, we define $f_{A} f d \mu:=\mu(A)^{-1} \int_{A} f d \mu$. A metric space $\left(X, d_{X}\right)$ is said to be geodesic if any two points $x, y \in X$ can be connected by a minimal geodesic between them, i.e., a rectifiable, constant speed curve $\gamma:[0, l] \longrightarrow X$ satisfying $\gamma(0)=x, \gamma(l)=y$, and length $(\gamma)=d_{X}(x, y)$. A subset $V \subset X$ is said to be convex if every two points in $V$ are joined by a minimal geodesic contained in $V$. Henceforth, let $\left(X, d_{X}, \mu\right)$ be a complete, geodesic metric measure space and assume the following.

Assumption 3.3. There exists an (at most) countable family of open sets in $X$, say $\left\{U_{n}\right\}_{n=1}^{\infty}$, which satisfies the following:

(1) $\overline{U_{n}} \subset U_{n+1}$ for all $n \geq 1$.

(2) $X=\bigcup_{n=1}^{\infty} U_{n}$. 
(3) Denote the connected components of $V_{n}:=\overline{U_{n}} \backslash U_{n-1}\left(=\left(U_{n} \backslash \overline{U_{n-1}}\right)^{-}\right)$by $\left\{V_{n, \alpha}\right\}_{\alpha=1}^{N_{n}}, 1 \leq N_{n} \leq \infty$, where we put $U_{0}:=\emptyset$. Then each $V_{n, \alpha}$ is convex and, for any $x \in X, R>0$, and any $n \geq 1$, only finitely many $V_{n, \alpha}$ 's intersect with $B(x, R)$.

(4) For any $x \in X, R>0$, and any $n \geq 1$, we have

$$
\limsup _{\varepsilon \rightarrow 0} \varepsilon^{-p} \mu\left(\left(B\left(U_{n}, \varepsilon\right) \backslash U_{n}\right) \cap B(x, R)\right)<\infty
$$

for a common $p \in(1, \infty)$, where $B\left(U_{n}, \varepsilon\right):=\left\{x \in X \mid \operatorname{dist}\left(x, U_{n}\right)<\varepsilon\right\}$.

(5) Each $\left(V_{n, \alpha}, d_{X}, \mu\right)$ satisfies the doubling condition and the weak Poincaré inequality of type $(1, p)$ for $p$ in (4).

In Assumption 3.3 (5), we need to treat not only balls contained in $V_{n, \alpha}$, but also the intersections of balls and $V_{n, \alpha}$, so that it requires the smoothness of the boundary of $V_{n, \alpha}$. We also remark that, by the doubling condition in (5) together with Assumption $3.3(3)$ and with the completeness, $\left(X, d_{X}\right)$ is proper. Therefore, $\mu$ is a Radon measure and (2) implies that, for any $x \in X$ and $R>0$, we have $B(x, R) \subset U_{n}$ for some $n$. (4) means that, roughly speaking, $\partial U_{n}$ has a codimension at least $p$ in $U_{n+1} \backslash U_{n}$. In particular, we have $\mu\left(\partial U_{n}\right)=0$. Therefore $X$ may have various dimensions.

Example 3.4. Let $X=\mathbb{R}^{m} \cup \mathbb{R}^{n} / \sim, m \leq n$ and $n \geq 2$, where $0_{\mathbb{R}^{m}} \sim 0_{\mathbb{R}^{n}}$. We consider the induced length metric and $\mu:=\left.\mathcal{L}^{m}\right|_{\mathbb{R}^{m}}+\left.\mathcal{L}^{n}\right|_{\mathbb{R}^{n}}$ on $X$, where $\mathcal{L}^{k}$ denotes the $k$-dimensional Lebesgue measure. Then $X$ satisfies Assumption 3.3 by putting $U_{1}=\mathbb{R}^{m} \backslash\left\{0_{\mathbb{R}^{m}}\right\}, U_{2}=X$, and $p=2$.

For $f \in L^{p}(X)$ and $x \in V_{n, \alpha}$, we set

$$
M(f)(x):=\sup _{0<r \leq R_{D}\left(V_{n, \alpha}\right) / 5} f_{B(x, r) \cap V_{n, \alpha}}|f| d \mu .
$$

The following two lemmas are proved in the standard ways (see $[\mathrm{HK}]$ and $[\mathrm{He}]$ ).

Lemma 3.5 (Maximal function theorem). Assume Assumption 3.3.

(i) For $f \in L^{1}(X)$ and $t>0$, we have

$$
\mu\left(\left\{x \in V_{n, \alpha} \mid M(f)(x)>t\right\}\right) \leq 2 C_{D}\left(V_{n, \alpha}\right) t^{-1}|f|_{L^{1}\left(\{|f| \geq t / 2\} \cap V_{n, \alpha}\right)} .
$$

(ii) For $f \in L^{p}(X)$, we have $|M(f)|_{L^{p}\left(V_{n, \alpha}\right)} \leq C_{1}\left(p, C_{D}\left(V_{n, \alpha}\right)\right)|f|_{L^{p}\left(V_{n, \alpha}\right)}$.

Lemma 3.6. Assume Assumption 3.3 and let $f \in H^{1, p}(X)$. For Lebesgue points $x, y \in V_{n, \alpha}$ of $f$ with $d_{X}(x, y) \leq \min \left\{R_{P}\left(V_{n, \alpha}\right) / 2, R_{D}\left(V_{n, \alpha}\right) / 10 \Lambda\left(V_{n, \alpha}\right)\right\}$, we have

$$
|f(x)-f(y)| \leq C_{2}\left(C_{D}, C_{P}\right) d_{X}(x, y)\left\{M\left(g_{f}^{p}\right)(x)^{1 / p}+M\left(g_{f}^{p}\right)(y)^{1 / p}\right\} .
$$

We define, as a 'core',

$$
\mathcal{C}:=\left\{f \in H^{1, p}(X) \cap C_{0}(X) \mid f \text { is locally Lipschitz on } U_{n} \backslash \overline{U_{n-1}} \text { for all } n \geq 1\right\},
$$

where $C_{0}(X)$ denotes the set of continuous functions on $X$ with compact supports.

For a continuous function $f: X \longrightarrow \mathbb{R}$ and a point $x \in X$, we define

$$
\operatorname{Lip} f(x):=\lim _{r \rightarrow 0} \sup _{y \in B(x, r) \backslash\{x\}} \frac{|f(x)-f(y)|}{d_{X}(x, y)} .
$$

Note that Lip $f$ is Borel measurable and, if $f$ is Lipschitz continuous, then it does not exceed the Lipschitz constant of $f$. It is not difficult to show that, for a locally Lipschitz function $f$, Lip $f$ is an upper gradient for $f([\mathrm{C}$, Proposition 1.11]). 
Theorem 3.7 ('Regularity'; cf. [C, Theorem 4.24]). Assume Assumption 3.3. Then the set $\mathcal{C}$ is dense in both $\left(H^{1, p}(X),|\cdot|_{H^{1, p}}\right)$ and $\left(C_{0}(X),|\cdot|_{\infty}\right)$.

Proof. The density in $\left(C_{0}(X),|\cdot|_{\infty}\right)$ is well known (see [He, Theorem 6.8]), so that it suffices to show that every function $f \in H^{1, p}(X)$ is approximated by a sequence of functions in $\mathcal{C}$ with respect to $|\cdot|_{H^{1, p}}$. It is not difficult to show that, by using Lemma 2.5. every $f \in H^{1, p}(X)$ can be approximated by bounded functions whose supports are bounded. Therefore, without loss of generality, we can suppose that $|f| \leq M$ for some $M>0$ and that supp $f \subset B\left(x_{0}, R\right)$ for some $x_{0} \in X$ and $R>0$. Furthermore, by Assumption 3.3(2), (3), and (4), we know supp $f \subset U_{N}$ for some $N \geq 1, \operatorname{supp} f \cap V_{n} \subset \bigcup_{\alpha=1}^{\alpha_{n}} V_{n, \alpha}$ for some $\alpha_{n}<\infty$, and

$$
C(n):=\limsup _{\varepsilon \rightarrow 0} \varepsilon^{-p} \mu\left(\left(B\left(U_{n}, \varepsilon\right) \backslash U_{n}\right) \cap B\left(x_{0}, R\right)\right)<\infty
$$

for all $n \geq 1$. Put

$$
C_{D}\left(V_{n}\right):=\max _{1 \leq \alpha \leq \alpha_{n}} C_{D}\left(V_{n, \alpha}\right), \quad R_{D}\left(V_{n}\right):=\min _{1 \leq \alpha \leq \alpha_{n}} R_{D}\left(V_{n, \alpha}\right),
$$

and define $C_{P}\left(V_{n}\right), R_{P}\left(V_{n}\right)$, and $\Lambda\left(V_{n}\right)$ in the same manner. In the remainder of this proof, we will omit ' $\cap B\left(x_{0}, R\right)$ ' for brevity.

We fix $n \geq 1$ for a while and consider an approximation of $\left.f\right|_{V_{n}}$. For $l \geq 1$, set

$$
A_{l}:=\left\{x \in V_{n} \mid \text { Lebesgue point of } f, M\left(g_{f}^{p}\right)(x) \leq l^{p}\right\} .
$$

Then, by Lemma 3.5(i), we find

$$
\mu\left(V_{n} \backslash A_{l}\right)=\mu\left(\left\{M\left(g_{f}^{p}\right)>l^{p}\right\} \cap V_{n}\right) \leq 2 C_{D}\left(V_{n}\right) l^{-p}\left|g_{f}\right|_{L^{p}\left(\left\{g_{f}^{p} \geq l^{p} / 2\right\} \cap V_{n}\right)}^{p},
$$

and hence $\lim _{l \rightarrow \infty} l^{p} \mu\left(V_{n} \backslash A_{l}\right)=0$. It follows from Lemma 3.6 that, for $x, y \in$ $A_{l} \cap V_{n, \alpha}$ with $\alpha \leq \alpha_{n}$ and $d_{X}(x, y) \leq \min \left\{R_{P}\left(V_{n}\right) / 2, R_{D}\left(V_{n}\right) / 10 \Lambda\left(V_{n}\right)\right\}$, we have

$$
|f(x)-f(y)| \leq 2 C_{2}\left(C_{D}, C_{P}\right) l d_{X}(x, y) .
$$

Since $V_{n, \alpha} \cap V_{n, \beta}=\emptyset$ if $\alpha \neq \beta$, we can choose a positive $\delta \leq \min \left\{R_{P} / 2, R_{D} / 10 \Lambda\right\} / 4$ for which $B\left(V_{n, \alpha}, 2 \delta\right) \cap B\left(V_{n, \beta}, 2 \delta\right)=\emptyset$ holds if $\alpha \neq \beta$ and $\alpha, \beta \leq \alpha_{n}$. Moreover, as $\overline{U_{n}} \subset U_{n+1}$ and $\overline{U_{n}} \cap B\left(x_{0}, R\right)^{-}$is compact, we can assume that $B\left(U_{n}, \delta\right) \subset U_{n+1}$ by taking smaller $\delta>0$ if necessary. Take $l$ large enough to satisfy $l^{-2}<\delta$ and

$$
\mu\left(V_{n} \backslash A_{l}\right)<\inf _{x \in V_{n} \cap B\left(x_{0}, R\right)} \mu\left(B(x, \delta) \cap V_{n}\right) .
$$

We remark that the right-hand side is positive by the doubling condition together with Assumption $3.3(3)$. We can extend $\left.f\right|_{A_{l}}$ to $W_{n, l}:=B\left(V_{n}, l^{-2}\right) \backslash \overline{U_{n-1}}$ by a local version of MacShane's lemma, more precisely,

$$
f_{n, l}(x):=\inf \left\{f(y)+2 C_{2} l d_{X}(x, y) \mid y \in A_{l} \cap B(x, 2 \delta)\right\} .
$$

Note that, for any $x \in W_{n, l}$, we have $\mu\left(A_{l} \cap B(x, 2 \delta)\right)>0$ by our construction and that, for any $y_{1}, y_{2} \in A_{l} \cap B(x, 2 \delta)$, we have $\left|f\left(y_{1}\right)-f\left(y_{2}\right)\right| \leq 2 C_{2} l d_{X}\left(y_{1}, y_{2}\right)$. Hence $f_{n, l}=f$ on $A_{l}$ and $f_{n, l}$ is locally Lipschitz on $W_{n, l}$ (with a Lipschitz constant $\left.2 C_{2} l\right)$. It follows from Assumption 3.3(4) that

$$
\lim _{l \rightarrow \infty} l^{p} \mu\left(W_{n, l} \backslash A_{l}\right)=\lim _{l \rightarrow \infty} l^{p}\left\{\mu\left(B\left(U_{n}, l^{-2}\right) \backslash U_{n}\right)+\mu\left(V_{n} \backslash A_{l}\right)\right\}=0,
$$


and hence, by Proposition 2.4.

$$
\begin{aligned}
& \left|f-f_{n, l}\right|_{H^{1, p}\left(W_{n, l}\right)} \\
& \leq\left|M+\left(M+4 C_{2} \delta l\right)\right|_{L^{p}\left(W_{n, l} \backslash A_{l}\right)}+\left|g_{f-f_{n, l}}\right|_{L^{p}\left(W_{n, l} \backslash A_{l}\right)} \\
& \leq\left(2 M+4 C_{2} \delta l\right) \mu\left(W_{n, l} \backslash A_{l}\right)^{1 / p}+\left|g_{f}\right|_{L^{p}\left(W_{n, l} \backslash A_{l}\right)}+2 C_{2} l \mu\left(W_{n, l} \backslash A_{l}\right)^{1 / p} \\
& \rightarrow 0
\end{aligned}
$$

as $l$ tends to infinity.

Fix $m \geq 1$ and define a partition of unity $\left\{\varphi_{n}\right\}_{n=1}^{\infty}$ by $\varphi_{0} \equiv 0$ and

$$
\varphi_{n}(x):=\left\{\begin{array}{cl}
1-\varphi_{n-1}(x) & \text { if } x \in \overline{U_{n}} \\
1-\left(\operatorname{dist}\left(x, U_{n}\right) l^{2}\right)^{1 / m} & \text { if } 0<\operatorname{dist}\left(x, U_{n}\right) \leq l^{-2} \\
0 & \text { otherwise }
\end{array}\right.
$$

for $n \geq 1$, inductively. Recall that $B\left(U_{n}, l^{-2}\right) \subset U_{n+1}$, and hence $\varphi_{n}$ is continuous. Note also that, by Assumption 3.3(1), $\sum_{n=1}^{\infty} \varphi_{n}=1$ holds on $B\left(x_{0}, R\right)$ if $l$ is sufficiently large. Set $f_{l}:=\sum_{n=1}^{\infty} \varphi_{n} f_{n, l}$. It is a finite sum since $\operatorname{supp} f$ is bounded, so that $f_{l} \in C_{0}(X)$ and $f_{l}$ is locally Lipschitz on each $U_{n} \backslash \overline{U_{n-1}}$. We have

$$
\left|f-f_{l}\right|_{H^{1, p}} \leq \sum_{n=1}^{\infty}\left|\varphi_{n}\left(f-f_{n, l}\right)\right|_{H^{1, p}\left(W_{n, l} \backslash A_{l}\right)}
$$

and

$$
\left|\varphi_{n}\left(f-f_{n, l}\right)\right|_{L^{p}\left(W_{n, l} \backslash A_{l}\right)} \leq\left(2 M+4 C_{2}\left(V_{n}\right) \delta l\right) \mu\left(W_{n, l} \backslash A_{l}\right)^{1 / p} \rightarrow 0,
$$

as $l$ tends to infinity.

We next estimate $\left|g_{\varphi_{n}\left(f-f_{n, l}\right)}\right|_{L^{p}\left(W_{n, l} \backslash A_{l}\right)}$. Let $\left\{\left(f_{i}, g_{i}\right)\right\}_{i=1}^{\infty}$ be a sequence such that $f_{i} \rightarrow f-f_{n, l}$ and $g_{i} \rightarrow g_{f-f_{n, l}}$ in $L^{p}\left(W_{n, l}\right)$ as $i \rightarrow \infty$, respectively, and that $g_{i}$ is an upper gradient for $f_{i}$. Clearly we may assume $\left|f_{i}\right| \leq 2 M+4 C_{2} \delta l$. Since $\varphi_{n}$ is bounded, $\varphi_{n} f_{i}$ tends to $\varphi_{n}\left(f-f_{n, l}\right)$ in $L^{p}\left(W_{n, l}\right)$ as $i \rightarrow \infty$. Hence it follows from Theorem 2.6 and Lemma 2.5 that

$$
\begin{aligned}
& \left|g_{\varphi_{n}\left(f-f_{n, l}\right)}\right|_{L^{p}\left(W_{n, l} \backslash A_{l}\right)} \\
& \leq \liminf _{i \rightarrow \infty}\left\{\left|\operatorname{Lip} \varphi_{n} \cdot\left(\left|f_{i}\right|+i^{-1}\right)\right|_{L^{p}\left(W_{n, l}\right)}+\left|\left(\varphi_{n}+i^{-1}\right) g_{i}\right|_{L^{p}\left(W_{n, l}\right)}\right\} \\
& \leq\left(2 M+4 C_{2} \delta l\right)\left|\operatorname{Lip} \varphi_{n}\right|_{L^{p}\left(W_{n, l}\right)}+\left|g_{f-f_{n, l}}\right|_{L^{p}\left(W_{n, l} \backslash A_{l}\right)} .
\end{aligned}
$$

On one hand, in the first part of this proof, we already observe that

$$
\lim _{l \rightarrow \infty}\left|g_{f-f_{n, l}}\right|_{L^{p}\left(W_{n, l} \backslash A_{l}\right)}=0 .
$$

On the other hand, we have

$$
\begin{aligned}
\left|\operatorname{Lip} \varphi_{n}\right|_{L^{p}\left(W_{n, l}\right)}^{p} & =\int_{0}^{\infty} \mu\left(\left\{\left(\operatorname{Lip} \varphi_{n}\right)^{p}>t\right\} \cap W_{n, l}\right) d t \\
& =\int_{0}^{\infty} p t^{p-1} \mu\left(\left\{\operatorname{Lip} \varphi_{n}>t\right\} \cap W_{n, l}\right) d t .
\end{aligned}
$$


It follows from $\left|(d / d s)\left(1-\left(s l^{2}\right)^{1 / m}\right)\right|=(1 / m) l^{2 / m} s^{(1-m) / m}$ together with Assumption 3.3(4) that

$$
\begin{aligned}
& \left|\operatorname{Lip} \varphi_{n}\right|_{L^{p}\left(W_{n, l}\right)}^{p} / p \\
& \leq \int_{l^{2} / m}^{\infty} t^{p-1}\left\{\mu\left(B\left(U_{n},\left(m t l^{-2 / m}\right)^{-m /(m-1)}\right) \backslash U_{n}\right)\right. \\
& \left.\quad \quad+\mu\left(B\left(U_{n-1},\left(m t l^{-2 / m}\right)^{-m /(m-1)}\right) \backslash U_{n-1}\right)\right\} d t \\
& \leq\{C(n-1)+C(n)\} \int_{l^{2} / m}^{\infty} t^{p-1}\left(m t l^{-2 / m}\right)^{-m p /(m-1)} d t \\
& =\{C(n-1)+C(n)\} m^{-m p /(m-1)} l^{2 p /(m-1)}\left[-\frac{m-1}{p} t^{-p /(m-1)}\right]_{l^{2} / m}^{\infty} \\
& =\{C(n-1)+C(n)\} \frac{m-1}{p} m^{-p}=\frac{C(n-1)+C(n)}{p} \frac{m-1}{m} m^{1-p} \\
& \rightarrow 0,
\end{aligned}
$$

as $m$ tends to infinity. Therefore we obtain $\left|f-f_{l}\right|_{H^{1, p}} \rightarrow 0$ as $m \rightarrow \infty$ and then $l \rightarrow \infty$. This completes the proof.

\section{INTRINSIC DISTANCE}

Theorem 3.7 allows us to adopt $\mathcal{C}$ as a set of test functions for defining the intrinsic distance according to Biroli and Mosco ( $[\mathrm{BM}]$ ).

Definition 4.1 (Intrinsic distance). For $p \in(1, \infty)$ and $x, y \in X$, define the $p$ intrinsic distance between $x$ and $y$ by

$$
d_{p}(x, y):=\sup \left\{f(x)-f(y) \mid f \in \mathcal{C}, g_{f, p} \leq 1 \text { a.e. on } X\right\} .
$$

We first recall Cheeger's theorem on the minimality of $\operatorname{Lip} f$ for a locally Lipschitz function $f$.

Theorem 4.2 ([C, Theorem 6.1]). Let $\left(X, d_{X}, \mu\right)$ be a complete metric measure space satisfying the doubling condition and the weak Poincaré inequality of type $(1, p)$ for some $p \in(1, \infty)$. Then, for any locally Lipschitz function $f \in H^{1, p}(X)$, we have $g_{f, p}=\operatorname{Lip} f$ a.e. on $X$. 2.4

The following is an immediate generalization of Theorem 4.2 through Proposition

Lemma 4.3. Let $\left(X, d_{X}, \mu\right)$ be a complete metric measure space satisfying Assumption 3.3 for some $p \in(1, \infty)$. Then, for any function $f \in H^{1, p}(X)$ which is locally Lipschitz on each $U_{n} \backslash \overline{U_{n-1}}$, we have $g_{f, p}=\operatorname{Lip} f$ a.e. on $X$.

Proposition 4.4. Let $\left(X, d_{X}, \mu\right)$ be a complete, geodesic metric measure space satisfying Assumption 3.3 for some $p \in(1, \infty)$. Then we have $d_{X} \leq d_{p}$ on $X$ and

$$
d_{p} \leq 2 C_{2}\left(C_{D}\left(V_{n, \alpha}\right), C_{P}\left(V_{n, \alpha}\right)\right) d_{X}
$$

on $V_{n, \alpha}$ for each $n, \alpha \geq 1$. Here $C_{2}$ is a constant in Lemma 3.6. In particular, $d_{p}$ gives the same topology on $X$ as $d_{X}$. 
Proof. Fix two points $x, y \in V_{n, \alpha}$ and a function $f \in \mathcal{C}$ with $g_{f, p} \leq 1$ a.e. on $X$. As $f$ is continuous, $x$ and $y$ are Lebesgue points of $f$, and hence it follows from Lemma 3.6 that $|f(x)-f(y)| \leq 2 C_{2} d_{X}(x, y)$.

Fix $x, y \in X$ and put $f(z):=\max \left\{d_{X}(x, y)-d_{X}(x, z), 0\right\}$ for $z \in X$. Then $f$ is 1-Lipschitz, $f \in \mathcal{C}$, and clearly $f(x)-f(y)=d_{X}(x, y)$. Therefore we obtain $d_{X}(x, y) \leq d_{p}(x, y)$.

\section{Strong Doubling CONDition And THE MAin THEOREM}

To improve the estimate $d_{X} \leq d_{p} \leq 2 C_{2} d_{X}$ in Proposition 4.4 to the equality $d_{p}=d_{X}$, we need a kind of measure contraction property. A measurable map $\Phi: X \times X \times[0,1] \longrightarrow X$ is called a geodesic bicombing if, for each $x, y \in X$, a map $[0,1] \ni t \longmapsto \Phi(x, y, t) \in X$ gives a minimal geodesic between $x$ and $y$.

Definition $5.1([\mathrm{R} 2])$. A geodesic metric measure space $\left(X, d_{X}, \mu\right)$ is said to satisfy the (local) strong doubling condition along a geodesic bicombing $\Phi: X \times X \times$ $[0,1] \longrightarrow X$ if there exist positive numbers $a=a(X)>0$ and $R=R(X)>0$ such that we have

$$
\mu(\Phi(x, A, t)) \geq a \mu(A)
$$

for any $x \in X, t \in[1 / 2,1]$, and any measurable subset $A \subset B(x, R)$.

Finite-dimensional Alexandrov spaces with lower curvature bounds as well as Riemannian manifolds with lower Ricci curvature bounds satisfy the strong doubling condition (see [KuSh, [R2]). Clearly the strong doubling condition implies the doubling condition. Furthermore, it is shown in R2 that, if $\left(X, d_{X}, \mu\right)$ satisfies the local strong doubling condition, then it satisfies the weak Poincaré inequality of type $(1,1)$ for the Cheeger-type energy form (see also [R1]).

Theorem 5.2. Let $\left(X, d_{X}, \mu\right)$ be a complete, geodesic metric measure space satisfying Assumption 3.3(1), (2), (3), and (4) for some $p \in(1, \infty)$. If, in addition, each $\left(V_{n, \alpha}, d_{X}, \mu\right)$ satisfies the strong doubling condition (along a geodesic bicombing $\left.\Phi=\Phi_{n, \alpha}\right)$, then we have $d_{X}=d_{p}$ on $X$.

Proof. Note that the strong doubling condition of $V_{n, \alpha}$ implies Assumption 3.3(5). We already know $d_{X} \leq d_{p}$ by Proposition 4.4, so we need only show $d_{p} \leq d_{X}$. We first show this inequality on $V_{n, \alpha}$. To do this, it is sufficient to prove that every $f \in \mathcal{C}$ with $g_{f, p} \leq 1$ a.e. on $X$ is 1-Lipschitz on $V_{n, \alpha}$. Suppose that there exist two distinct points $x, y \in V_{n, \alpha}$ and a function $f \in \mathcal{C}$ with $g_{f, p} \leq 1$ a.e. on $X$ such that we have $|f(x)-f(y)| \geq(1+2 \varepsilon) d_{X}(x, y)$ for some $\varepsilon>0$. Since $V_{n, \alpha}$ is convex, without loss of generality, we may assume $d_{X}(x, y) \leq R\left(V_{n, \alpha}\right) / 2$. We remark that $\left.f\right|_{V_{n, \alpha}}$ is locally Lipschitz by Lemma 3.6. Since $f$ is continuous, we can find a sufficiently small $r>0$ such that $|f(w)-f(z)| \geq(1+\varepsilon) d_{X}(w, z)$ holds for all $w \in B(x, r) \cap V_{n, \alpha}$ and $z \in B(y, r) \cap V_{n, \alpha}$. We define $A:=\left\{z \in V_{n, \alpha} \mid \operatorname{Lip} f(z) \geq 1+\varepsilon\right\}$, denote by $\chi_{A}$ the characteristic function of $A$, and set $k$ as a smallest integer not smaller than $-\log _{2} r$. We put $\Phi_{0}:=\Phi$ and $\Phi_{i}(x, z, t):=\Phi\left(x, \Phi_{i-1}(x, z, 1 / 2), t\right)$ for $i=1,2, \ldots, k-1$, inductively. Then we have, for every $z \in B(y, r) \cap V_{n, \alpha}$,

$$
\sum_{i=0}^{k-1} \int_{1 / 2}^{1} \chi_{A}\left(\Phi_{i}(x, z, t)\right) d t>0,
$$


since $\left|f\left(\Phi_{k-1}(x, z, 1 / 2)\right)-f(z)\right| \geq(1+\varepsilon) d_{X}\left(\Phi_{k-1}(x, z, 1 / 2), z\right)$. Note also that $\mu\left(B(y, r) \cap V_{n, \alpha}\right)>0$. By the strong doubling condition, we obtain

$$
\begin{aligned}
0 & <\sum_{i=0}^{k-1} \int_{B(y, r) \cap V_{n, \alpha}} \int_{1 / 2}^{1} \chi_{A}\left(\Phi_{i}(x, z, t)\right) d t d \mu(z) \\
& =\sum_{i=0}^{k-1} \int_{1 / 2}^{1} \int_{B(y, r) \cap V_{n, \alpha}} \chi_{A}\left(\Phi_{i}(x, z, t)\right) d \mu(z) d t \\
& =\sum_{i=0}^{k-1} \int_{1 / 2}^{1} \int_{\Phi_{i}\left(x, B(y, r) \cap V_{n, \alpha}, t\right)} \chi_{A}(z)\left(\Phi_{i}(x, \cdot, t)_{*}(d \mu)\right)(z) d t \\
& \leq \sum_{i=0}^{k-1} a\left(V_{n, \alpha}\right)^{-(i+1)} \int_{1 / 2}^{1} \int_{\Phi_{i}\left(x, B(y, r) \cap V_{n, \alpha}, t\right)} \chi_{A}(z) d \mu(z) d t .
\end{aligned}
$$

Therefore we have $\mu(A)>0$, but it is a contradiction. Thus every $f \in \mathcal{C}$ with $g_{f, p} \leq 1$ a.e. on $X$ is 1-Lipschitz on $V_{n, \alpha}$, so that we obtain $d_{p}=d_{X}$ on $V_{n, \alpha}$.

For general $x \in V_{n, \alpha}$ and $y \in V_{m, \beta}$, let $\gamma:\left[0, d_{X}(x, y)\right] \longrightarrow X$ be a minimal geodesic between them. By Assumption 3.3. (2) and (3), we have

$$
\gamma\left(\left[0, d_{X}(x, y)\right]\right) \subset \bigcup_{k=1}^{N} \bigcup_{\sigma=1}^{N} V_{k, \sigma}
$$

for some $N \geq 1$. Set $t_{0}:=0,\left(k_{0}, \sigma_{0}\right):=(n, \alpha)$, and

$$
t_{1}:=\sup \left\{t \in\left[0, d_{X}(x, y)\right] \mid \gamma(t) \in V_{k_{0}, \sigma_{0}}\right\} .
$$

If $t_{1}=d_{X}(x, y)$, then we put $\left(k_{1}, \sigma_{1}\right):=(m, \beta)(=(n, \alpha))$. If not, then we find $\gamma\left(t_{1}\right) \in V_{k^{\prime}, \sigma^{\prime}}$ for some $\left(k^{\prime}, \sigma^{\prime}\right) \neq\left(k_{0}, \sigma_{0}\right)$, and put $\left(k_{1}, \sigma_{1}\right):=\left(k^{\prime}, \sigma^{\prime}\right)$ and $t_{2}:=$ $\sup \left\{t \in\left[t_{1}, d_{X}(x, y)\right] \mid \gamma(t) \in V_{k_{1}, \sigma_{1}}\right\}$. We iterate this construction and obtain a sequence $0=t_{0}<t_{1}<t_{2}<\cdots<t_{M}=d_{X}(x, y)$. By our construction, we observe $M \leq N^{2}$. By the first part of this proof, we know $d_{p}\left(\gamma\left(t_{l-1}\right), \gamma\left(t_{l}\right)\right)=$ $d_{X}\left(\gamma\left(t_{l-1}\right), \gamma\left(t_{l}\right)\right)$ for $l=1,2, \ldots, M$, and hence we conclude that

$$
\begin{aligned}
d_{p}(x, y) & \leq \sum_{l=1}^{M} d_{p}\left(\gamma\left(t_{l-1}\right), \gamma\left(t_{l}\right)\right)=\sum_{l=1}^{M} d_{X}\left(\gamma\left(t_{l-1}\right), \gamma\left(t_{l}\right)\right) \\
& =d_{X}(x, y) .
\end{aligned}
$$

This completes the proof.

In a quite general setting, the condition $\operatorname{Lip} f \leq 1$ a.e. does not imply the 1Lipschitz continuity of a Lipschitz function $f$. At least, the Poincaré inequality is necessary to ensure that, if $f$ has zero energy, then it is constant.

\section{Distinguish METRIC SPACES By ENERGy FORMS}

Two distance functions $d$ and $d^{\prime}$ on a space $X$ are said to be equivalent if there exists a constant $C \geq 1$ for which $C^{-1} d \leq d^{\prime} \leq C d$ holds on $X$. By Theorem 5.2 with $X=V_{1,1}$, we find the following.

Corollary 6.1. Let $d$ and $d^{\prime}$ be two equivalent distance functions on a measure space $(X, \mu)$ such that both $(X, d)$ and $\left(X, d^{\prime}\right)$ are complete and geodesic and that 
both $(X, d, \mu)$ and $\left(X, d^{\prime}, \mu\right)$ satisfy the strong doubling condition. If $g_{f, p}^{d}=g_{f, p}^{d^{\prime}}$ holds a.e. on $X$ for some $p \in(1, \infty)$ and every $f \in \mathcal{C}$, then we have $d=d^{\prime}$.

Here we denote by $g_{f, p}^{d}$ and $g_{f, p}^{d^{\prime}}$ the minimal generalized upper gradients for $f$ with respect to $d$ and $d^{\prime}$, respectively. We remark that the core $\mathcal{C}$ defined as (3.1) is common to $d$ and $d^{\prime}$ since they are equivalent.

Corollary 6.1 means that we can distinguish two (equivalent) distance functions by comparing energy measures of functions in the core $\mathcal{C}$. In the reminder of this article, we shall observe that, if we consider the Korevaar-Schoen-type energy form in place of the Cheeger-type, then we cannot distinguish some metric spaces. In particular, we cannot reconstruct the distance function by using the KorevaarSchoen-type energy form. In the following, we treat only the case of $p=2$.

Definition 6.2 (Korevaar-Schoen-type energy form). For $f \in L^{2}(X)$, define the Korevaar-Schoen-type 2-energy $E_{2}^{K S}(f)$ of $f$ by

$$
E_{2}^{K S}(f):=\limsup _{r \rightarrow 0} \int_{X}\left\{\int_{B(x, r) \backslash\{x\}} \frac{|f(x)-f(y)|^{2}}{r^{2}} \frac{d \mu(y)}{\mu(B(y, r))^{1 / 2}}\right\} \frac{d \mu(x)}{\mu(B(x, r))^{1 / 2}} .
$$

This definition is due to $[\underline{\mathrm{S}}$ and is slightly different from that in $\mathrm{KoSc}$. By $[\underline{\mathrm{S}}$, Theorem 5.6], $E_{2}^{K S}$ gives a Dirichlet form if $\left(X, d_{X}, \mu\right)$ satisfies the strong Measure Contraction Property.

Example 6.3. Let $n \geq 2$ and $\left(X, d_{X}, \mu\right)=\left(\mathbb{R}^{n},|\cdot|, d x\right)$ be an $n$-dimensional Banach space with the standard measure. Then there exists an inner product $\langle\cdot, \cdot\rangle$ on $\mathbb{R}^{n}$ such that we have $E_{2}^{K S}(f)=\int_{\mathbb{R}^{n}}\langle d f, d f\rangle d x$ for every $f \in C_{0}^{\infty}\left(\mathbb{R}^{n}\right)$. On the other hand, we know $E_{2}^{C}(f)=\int_{\mathbb{R}^{n}}|d f|_{*}^{2} d x$, where we denote by $\left(\mathbb{R}^{n},|\cdot|_{*}\right)$ the dual space of $\left(\mathbb{R}^{n},|\cdot|\right)$.

Note that the moduli space consisting of inner products on $\mathbb{R}^{n}$, that is, the space of positive definite symmetric $n \times n$ matrices, is obviously finite dimensional. However, the moduli space of norms on $\mathbb{R}^{n}$ is infinite dimensional, so that many norms give the same Korevaar-Schoen-type energy form. For example, for all $l_{p^{-}}$ norm $|\cdot|_{p}$ with $p \in[1, \infty]$, the associated inner product is a constant multiple of the standard Euclidean one (because they are symmetric enough). If we denote that constant by $c_{p}$, then the Korevaar-Schoen-type energy form with respect to the norm $c_{p}^{1 / 2}|\cdot|_{p}$ coincides with the standard Dirichlet form on $\mathbb{R}^{n}$. Therefore the Korevaar-Schoen-type energy form cannot distinguish these (uncountably many) norms.

\section{ACKNOWLEDGEMENT}

I wish to express my gratitude to Professor Takashi Shioya for his valuable comments.

\section{REFERENCES}

[BM] M. Biroli and U. Mosco, A Saint-Venant type principle for Dirichlet forms on discontinuous media, Ann. Mat. Pura Appl. 169 (1995), 125-181. MR1378473 (97b:35082)

[C] J. Cheeger, Differentiability of Lipschitz functions on metric measure spaces, Geom. Funct. Anal. 9 (1999), 428-517. MR.1708448 (2000g:53043)

[FOT] M. Fukushima, Y. Oshima, and M. Takeda, Dirichlet forms and symmetric Markov processes, de Gruyter Studies in Mathematics 19, Walter de Gruyter \& Co., Berlin, 1994. MR 1303354(96f:60126) 
[HK] P. Hajłasz and P. Koskela, Sobolev met Poincaré, Mem. Amer. Math. Soc. 145 (2000), no. 688. MR 1683160 (2000j:46063)

[He] J. Heinonen, Lectures on analysis on metric spaces, Universitext, Springer-Verlag, New York, 2001. MR 1800917 (2002c:30028)

[HeK] J. Heinonen and P. Koskela, Quasiconformal maps in metric spaces with controlled geometry, Acta Math. 181 (1998), 1-61. MR.1654771 (99j:30025)

[KoSc] N. J. Korevaar and R. M. Schoen, Sobolev spaces and harmonic maps for metric space targets, Comm. Anal. Geom. 1 (1993), 561-659. MR.1266480 (95b:58043)

[KMS] K. Kuwae, Y. Machigashira, and T. Shioya, Sobolev spaces, Laplacian, and heat kernel on Alexandrov spaces, Math. Z. 238 (2001), 269-316. MR1865418(2002m:58052)

[KuSh] K. Kuwae and T. Shioya, On generalized measure contraction property and energy functionals over Lipschitz maps, Potential Anal. 15 (2001), 105-121. MR1838897 (2002f:31022)

[O] S. Ohta, Regularity of harmonic functions in Cheeger-type Sobolev spaces, Ann. Global Anal. Geom. 26 (2004), 397-410. MR2103408(2005h:35053)

[R1] A. Ranjbar-Motlagh, A note on the Poincaré inequality, Studia Math. 154 (2003), 1-11. MR 1949045 (2004b:46039)

[R2] A. Ranjbar-Motlagh, Poincaré inequality for abstract spaces, Bull. Austral. Math. Soc. 71 (2005), 193-204. MR2133404

[S] K.-T. Sturm, Diffusion processes and heat kernels on metric spaces, Ann. Probab. 26 (1998), 1-55. MR1617040 (99b:31008)

Department of Mathematics, Faculty of Science, Kyoto University, Kyoto 606-8502, JAPAN

E-mail address: sohta@math.kyoto-u.ac.jp 